Brit. Heart F., 1966, 28, 244.

\title{
Genesis and Evolution of Ectopic Ventricular Rhythm
}

\author{
L. SCHAMROTH
}

From the Baragwanath Hospital and University of the Witwatersrand, fohannesburg, Republic of South Africa

Not chaos-like together, crush'd and bruis'd, But, as the world, harmoniously confused:

Where order in variety we see,

And where, tho' all things differ, all agree.

$$
\text { Alexander Pope, Windsor Forest. }
$$

Ectopic ventricular rhythm is represented by ventricular escape rhyth.m, ventricular extrasystoles, ventricular parasys.o'e, ventricular tachycardia, and ventricular fibrillation. The relation between these forms of ectopic ventricular rhythm is at present ill defined with but a few tenuous links. Thus, it is known that the same ectopic focus that gives rise to a parasystolic rhythm can also produce extrasystoles; and ventricular tachycardia may be regarded as a succession of consecutive ventricular extrasystoles; it has also been observed that a very rapid ventricular tachycardia frequently precedes ventricular fibrillation. More recently, the recognition of the phenomenon of concealed extrasystoles (Schamroth and Marriott, 1961, 1963) and its relation to parasystole has revealed new characteristics of extrasystoles-characteristics that provide an essential link in the evolutionary chain of ectopic ventricular rhythm.

It is the purpose of this communication to analyse the available evidence for properties common to the various forms of ectopic ventricular rhythm, to record new observations on the problem, and to formulate a unifying concept of ectopic ventricular genesis and its development in the light of these observations and recent cardiophysiological developments.

\section{Physiological Considerations}

Transmembrane Potential of Pacemaking and Nonpacemaking Cells. The transmembrane potential of pacemaking and non-pacemaking cells differs in one fundamental respect. Non-pacemaking cells have a stable resting diastolic potential, i.e. a resting

\footnotetext{
Received January 18, 1965.
}

potential that remains at a constant subthreshold or "horizontal" level until depolarization by a propagated impulse results in abrupt reversal to the action potential (Fig. 1A). Pacemaking cells, however, have an unstable diastolic potential which exhibits a gradual upwards slope of slow diastolic depolarization until the level of instability-the threshold potential-is reached; there is then a smooth transition to the upstroke of the action potential (Fig. 1B)-(Draper and Weidmann, 1951).

On this basis, there are three variable factors that determine the exact moment the unstable diastolic depolarizing potential of a pacemaking cell reaches threshold level, and that in turn determine the discharge frequency of a pacemaking cell.

(1) Variations in the angle of the slope of spontaneous diastolic depolarization. If the slope becomes steeper it requires less time to reach threshold level and the rate increases (illustrated in Fig. 1C by a change from the solid line A to the dotted line B).

(2) Variations in the level of the threshold potential. The rate increases with a lowered threshold level as less time is then required for the slope of spontaneous diastolic depolarization to reach the threshold level (illustrated in Fig. 1D by a change from the solid line A to the dotted line B).

(3) Variations in the level of the resting potential. Variations in the relationship of the resting potential to the threshold level will affect the rate on a similar basis to that described under (2). With a greater or "deeper" resting level, it will take longer for the slope of spontaneous diastolic depolarization to reach threshold level and the rate slows; conversely, with a "shallower" slope the rate accelerates.

Most, if not all, Purkinje fibres have potential pacemaking ability, and it is very likely that all spontaneous activity of ventricular myocardial origin arises in the terminal twigs of the Purkinje system (Hoffman and Cranefield, 1960). 


\section{OBSERVATIONS ON VARIOUS Forms OF VeNTRICULAR RHYTHM \\ Ventricular Escape Rhythm}

The ventricular escape beat is the simplest form of ectopic ventricular rhythm. It occurs whenever the sinus impulse fails to reach the ventricles: this failure may be due to marked sinus bradycardia, sino-atrial- or atrio-ventricular block. The escape beat always occurs at a constant interval from the preceding sinus beat; when more than one sinus or supraventricular impulse fails to reach the ventricles, the escape focus continues to discharge regularly at the same escape interval resulting in an idioventricular escape rhythm. This escape rhythm continues until a subsequent sinus impulse reaches the ventricles and abolishes the ectopic discharge. These principles are illustrated in Cases 1,2, and 3.

Case 1. Fig. 2 illustrates ventricular escape beats during atrial fibrillation. The bizarre QRS complexes of the escape beats are seen in the upper strip-second and third QRS complexes; and in the lower strip-second QRS complex. These complexes occur at a near-constant interval of 152-156* from the preceding conducted beats; the ectopic cycle length of the escape rhythm (upper strip) is the same as that of the escape interval.

Case 2. Fig. 3 illustrates ventricular escape following the blocked sinus impulse of an A-V nodal Wenckebach conduction sequence. The bizarre QRS complexes of the escaping focus follow the preceding conducted beat at a constant interval of 74. In two instances in the lower strip the escape rhythm continues for 2 beats at the same escape interval of 74 (1st and 2nd, and 4th and 5th bizarre complexes; the 5th complex is a fusion beat). Subsequent escapes are prevented by the conducted sinus impulses.

Case 3. Fig. 4 illustrates idioventricular escape rhythm following the precipitation of sinus brady-

$\star$ All time intervals are given in hundredths of a second, i.e. $152=152$ hundredths of a second.
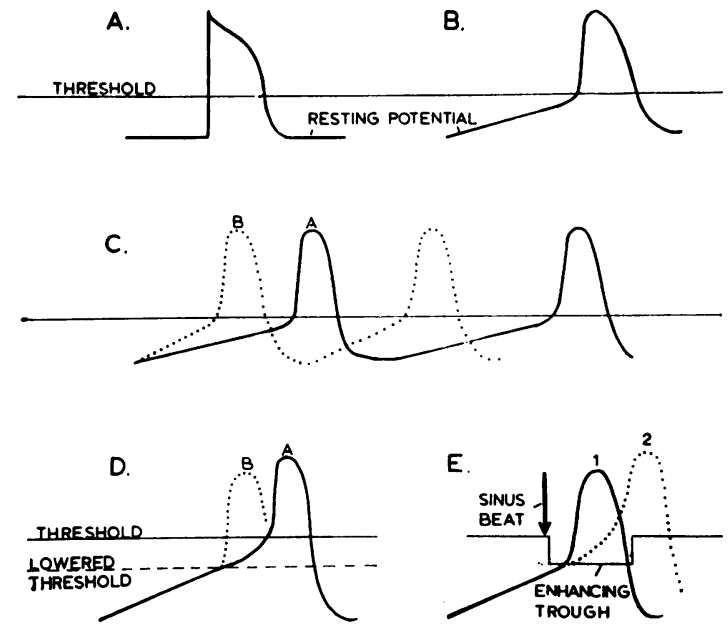

Fig. 1.-Diagrammatic representation of: (A) the transmembrane potential of a non-pacemaking cell; (B) the transmembrane potential of a pacemaking cell; $(C)$ the effect of a steeper diastolic depolarizing slope on the cardiac rate; (D) the effect of lowered threshold on the heart rate; and (E) the mechanism of precipitation of an extrasystole. (See text.)

cardia by eyeball compression (applied in the interval between arrows). The bizarre QRS complexes represent the idioventricular escape rhythm occurring at an escape interval of 128 . The ectopic cycle length of the escape rhythm is also 128 . The second last complex in the second strip is normal in configuration and represents a conducted sinus beat. Following this, the ectopic rhythm recommences (with a fusion beat) after the same interval of 128 . (The short paroxysm of ventricular tachycardia in the second strip is discussed in the section on ventricular tachycardia.)

The aforementioned features indicate that the ventricular escape focus has two fundamental properties.

(1) The focus is pacemaking as indicated by (a) the fixed generation time of the escape beat; and (b) the regular discharge of the idioventricular escape rhythm with the same cycle as the escape interval.

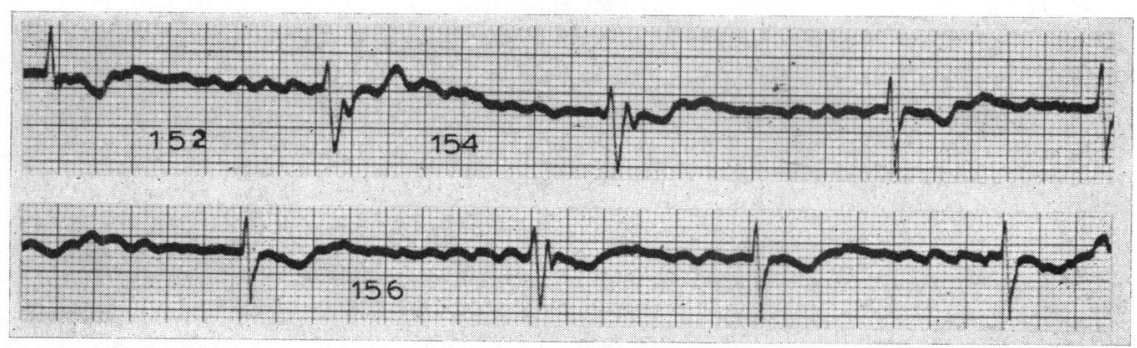

FIG. 2.-Electrocardiogram (continuous strip) showing ventricular escape rhythm during atrial fibrillation. 


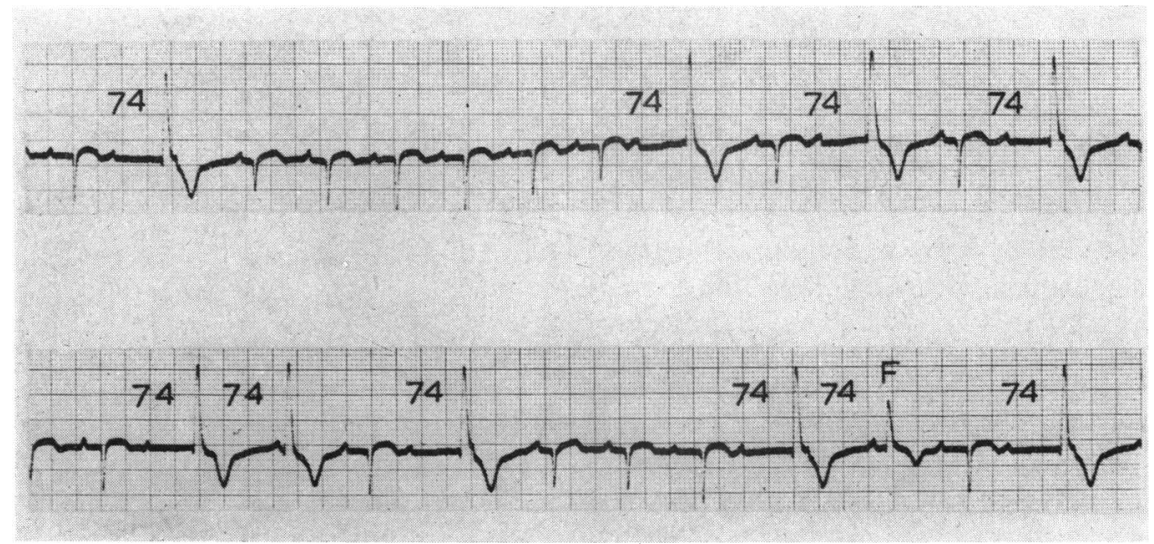

Fig. 3.-Electrocardiogram (continuous strip) showing ventricular escape beats following the blocked impulses of A-V Wenckebach conduction sequences.

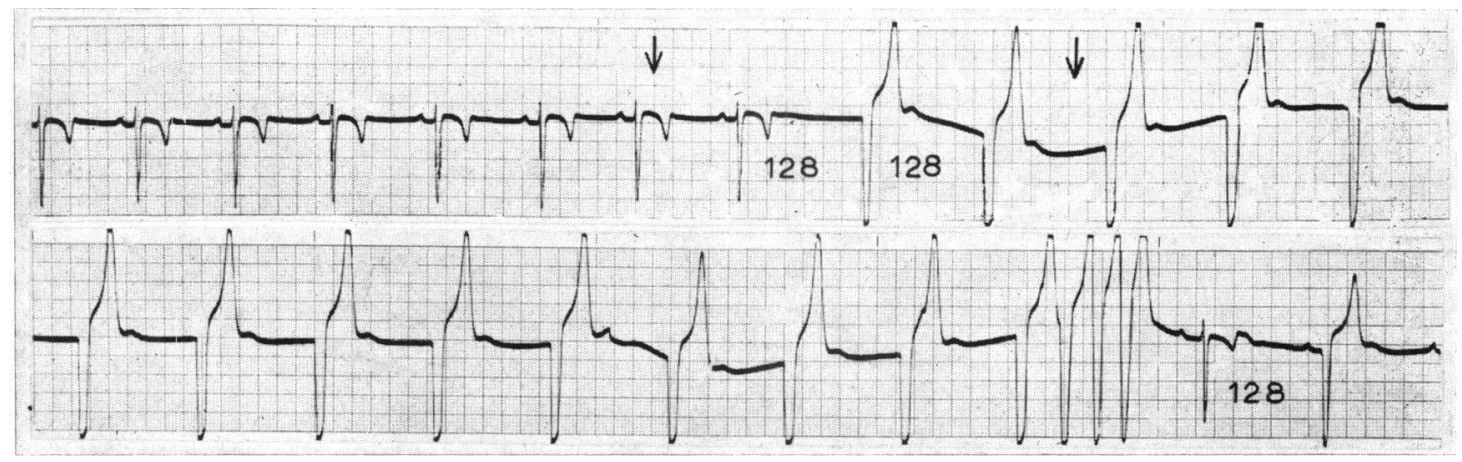

FIG. 4.-Electrocardiogram (continuous strip) showing idioventricular escape rhythm following eyeball compression (applied during the interval indicated by the arrows).

(2) The ectopic focus is not protected from the dominant pacemaker as indicated by the abolition of its discharge if and when the sinus impulse reaches it.

\section{Ventricular Parasystole}

In ventricular parasystole an ectopic ventricular focus discharges regularly and is undisturbed by the dominant pacemaker, despite the fact that the dominant impulse is able to activate the surrounding myocardium. The regular ectopic discharge becomes manifest whenever it finds the surrounding myocardium non-refractory following excitation by the dominant pacemaker. Thus the sinus and parasystolic rhythms discharge independently and asynchronously, resulting in: (1) varying coupling intervals (the coupling interval is the interval between the ectopic beat and the preceding sinus beat); (2) long interectopic intervals which are in simple multiples of the shorter interectopic intervals; (3) fusion beats due to coincident activation of the ventricles by the parasystolic and sinus impulses. These features are illustrated in Case 4.

Case 4. Fig. 5 shows sinus rhythm interspersed with bizarre QRS complexes of an ectopic ventricular discharge. These bizarre QRS complexes have varying coupling intervals; the long interectopic intervals are in simple multiples of 135 $( \pm 1 \cdot 5)$. A fusion beat is present at the end of strip 2 (labelled " $F$ "). These features indicate the presence of ventricular parasystole.

The aforementioned features indicate that a parasystolic focus has two fundamental properties.

(1) The ectopic focus is a pacemaking centre as indicated by its regular independent discharge. 


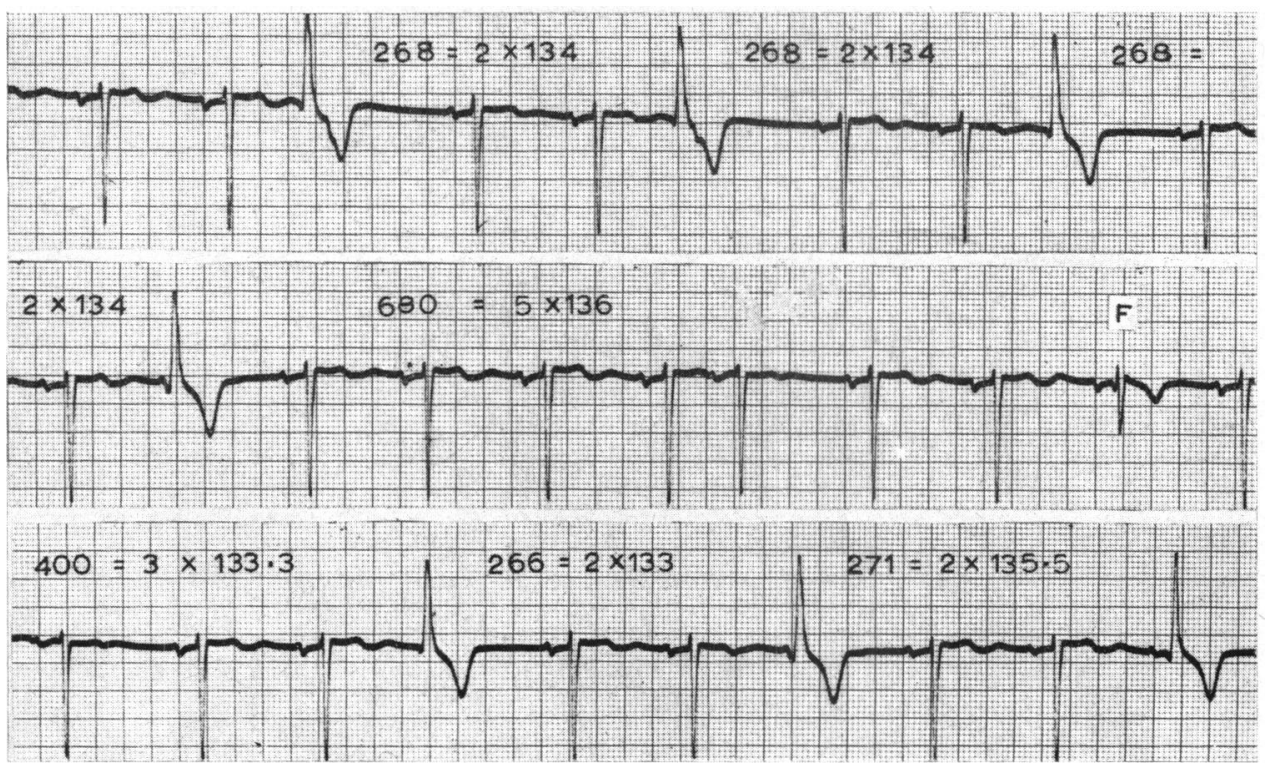

Fig. 5.-Electrocardiogram (continuous strip) of Case 4 showing parasystole. $F=$ fusion beat.

(2) The ectopic focus is protected in its immediate vicinity from all other impulses and this protection is operative throughout the duration of the ectopic cycle, i.e. during both its refractory and nonrefractory phases (Schamroth, 1964).

Occasionally a parasystolic discharge does not become manifest even if its timing is such that it occurs during the non-refractory phase of the ventricular cycle, i.e. calculation shows that the ectopic focus discharges but does not invade the surrounding myocardium, even though the myocardium is responsive at the time. This phenomenon is explained by the presence of exit blockAustrittsblockierung-at the ectopic ventricular junction. For example, in the presence of a $4: 1$ exit block, only 1 in every 4 ectopic discharges becomes manifest. The presence of exit block thus masks a rapid ectopic discharge. Exit block may be of a high grade; in the case described by Schamroth (1962) a ventricular parasystolic rhythm was operative with a varying $7: 1,8: 1$, and $9: 1$ exit block. The true ectopic rate was thus 7,8 , or 9 times faster than apparent and, in fact, represented an ectopic tachycardia.

\section{Ventricular Extrasystoles}

(1) Coupling of Extrasystoles. Ventricular extrasystoles are characterized by constant coupling intervals, i.e. the interval between the preceding sinus beat and the extrasystole is constant for all extra- systoles of the same shape in the same tracing. This indicates that the extrasystole is in some way associated with, dependent upon, or precipitated by, the preceding sinus beat. There are two prevailing concepts of this relationship: (a) the re-entry theory; and (b) the theory of ectopic enhancement.

The re-entry theory assumes a localized area of refractoriness in the vicinity of the ectopic focus. The sinus impulse is unable to penetrate the ectopic focus but, after depolarizing the surrounding tissue, approaches the previously refractory area from another direction. The focus has meanwhile become excitable again and is therefore able to propagate the re-entering impulse thereby initiating an extrasystole.

The theory of ectopic enhancement assumes that the extrasystole is generated as a result of some enhancing effect on a subthreshold focus by the preceding sinus beat. There are several such enhancing mechanisms which could conceivably play a role, the most likely being the phenomenon of Wedensky facilitation. Wedensky (1903) showed that an impulse arriving at a blocked zone enhanced the excitability beyond the block; this may be brought about by either a diminution in the resting potential, or, more likely, a lowering of threshold. Thus, if there is some local impediment to the conduction of the sinus impulse to the ectopic focus, the threshold of the ectopic focus is lowered and its excitability enhanced so that it may then initiate its own impulse-the extrasystole. These theories are considered further (see below). 


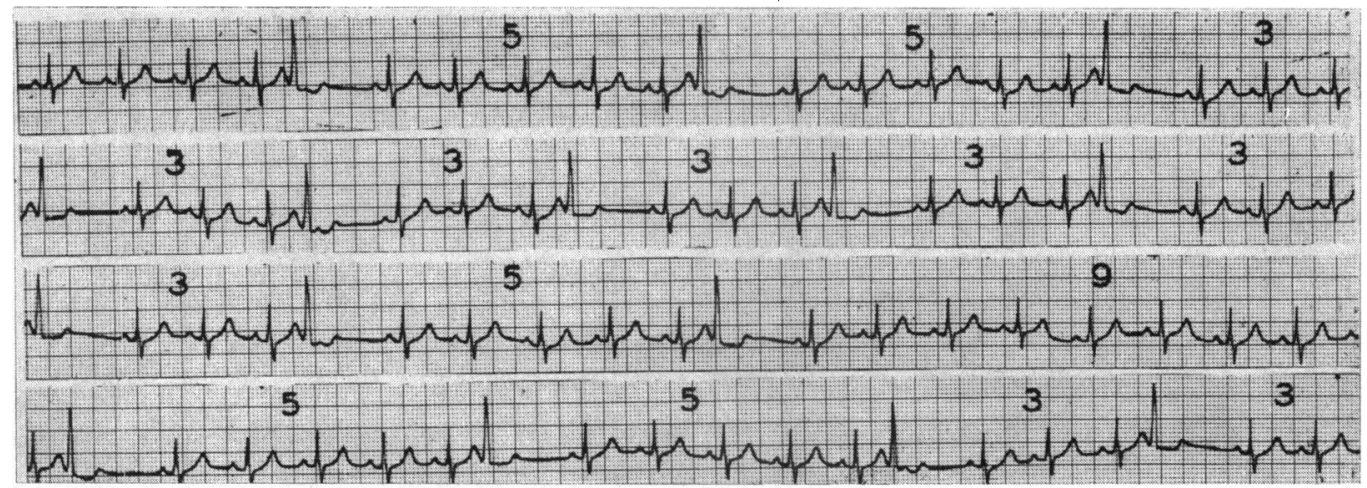

FIG. 6.-Electrocardiogram (continuous strip), showing concealed bigeminy. There are only odd numbers of sinus beats between the manifest extrasystoles. (See also Fig. 11.)

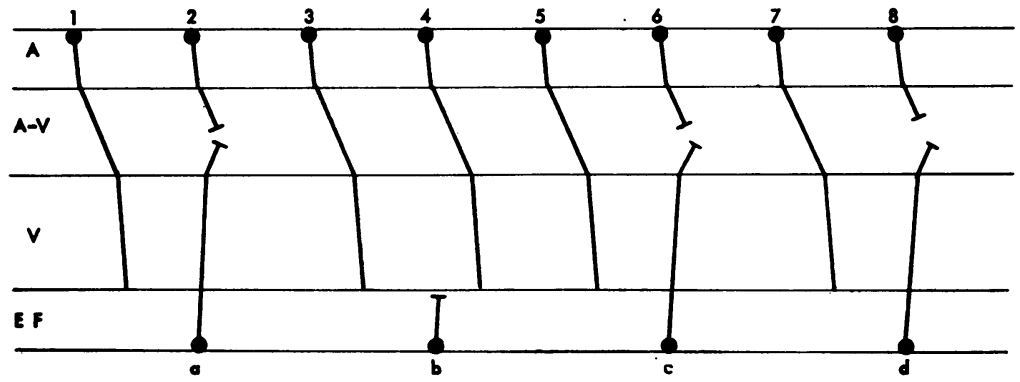

FIG. 7.-Diagrammatic representation of concealed bigeminy. (See text.)

(2) Distribution of Ventricular Extrasystoles. Ventricular extrasystoles are frequently distributed in well-defined and regular rhythmic patterns. For example, they may be found after every alternate sinus beat resulting in bigeminal rhythm, or, after every second sinus beat resulting in a form of trigeminal rhythm. They may also occur in random distribution. Recently, however, it has been shown that even a random distribution may mask an underlying regularity. Analysing long continuous recordings of ventricular extrasystoles in apparent random distribution, Schamroth and Marriott $(1961,1963)$ observed that, in many instances, there were only odd numbers of sinus beats between extrasystoles. This phenomenon is illustrated in Case 5.

Case 5. Fig. 6 is a section of a 15-minute continuous recording and shows only odd numbers of sinus beats between the extrasystoles. Longer interectopic intervals (not illustrated) containing $37,51,23,17$, and 43 intervening sinus beats were also observed in the same recording. No even numbers were seen.
Many examples have been noted since the original observation. This phenomenon was explained on the basis that a persistent and continuous bigeminal rhythm was present but that some of the ventricular extrasystoles remained localized to the ectopic focus without invading the surrounding myocardium - a condition analogous to the exit block of parasystole. The situation is schematically represented in Fig. 7. A normally occurring extrasystole is represented by " $a$ " which prevents the descent of sinus beat 2 . In ordinary bigeminal rhythm the next coupled extrasystole would occur $a t$ " $b$ " and the next at " $c$ ". If, however, the second extrasystole " $b$ " remains confined to its focus and fails to invade the myocardium (as depicted at " $\forall$ "), then the descent of sinus beat 4 will not be prevented and three sinus beats (labelled 3, 4 , and 5) will appear between the extrasystoles " $a$ " and " $c$ ". If beat " $c$ " were also confined to its focus, sinus beats 6 and 7 would be included between extrasystoles " $a$ " and " $d$ ", and this would leave five sinus beats (labelled 3 to 7 ) between these manifest extrasystoles. Thus, if the bigeminal discharges are uninterrupted but one or more of 


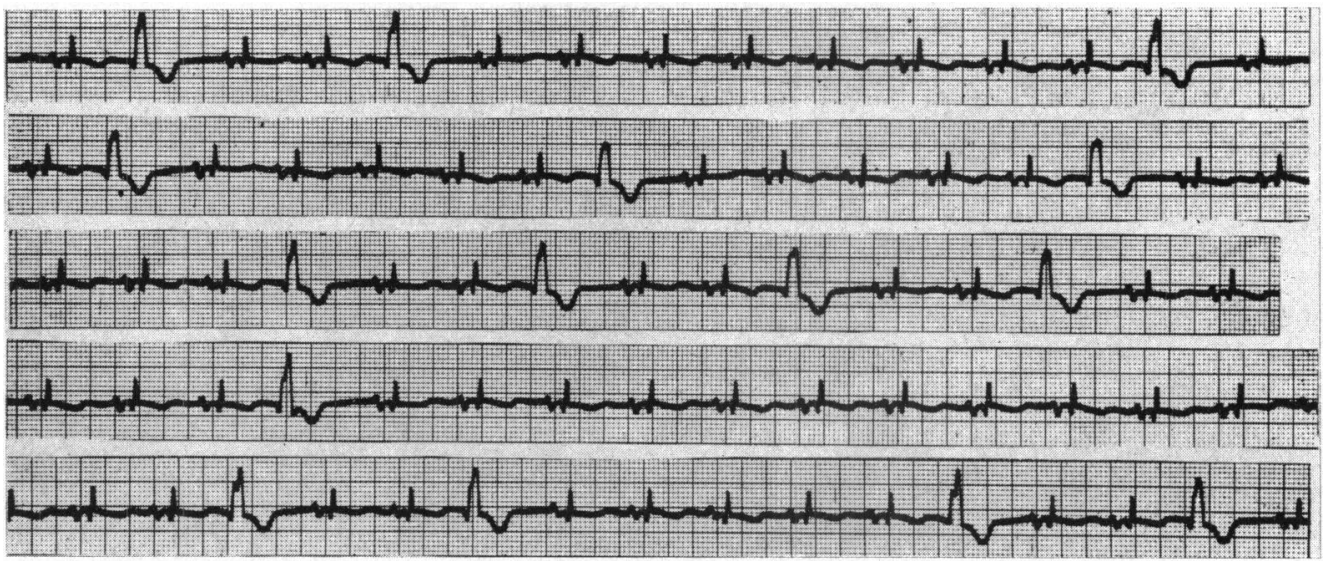

FIG. 8.-Electrocardiogram (continuous strip) showing concealed trigeminy. The sinus beats between extrasystoles are only in numbers of $2,5,8,11$, and 14 . (See text.)

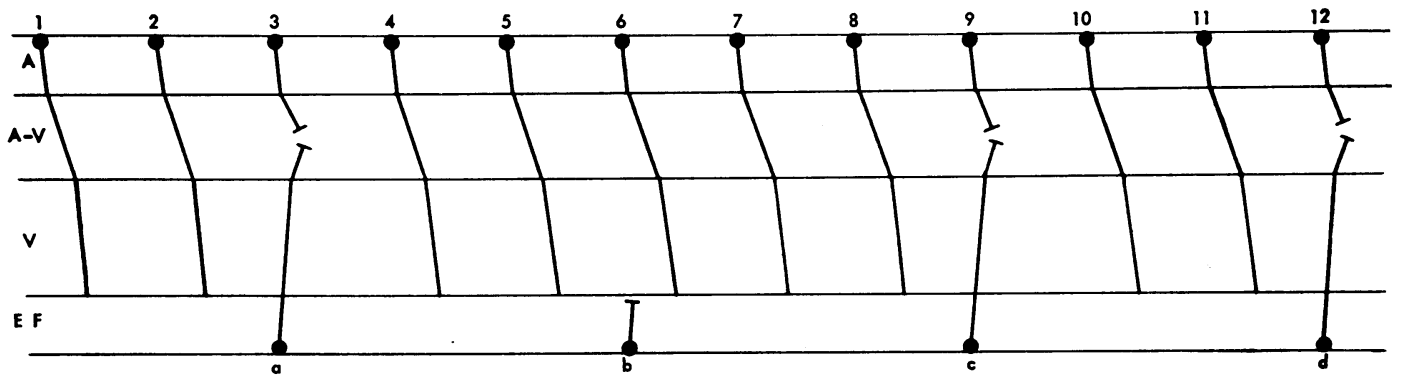

FIG. 9.-Diagrammatic representation of concealed trigeminy. (See text.)

them fails to invade the myocardium, an odd number of sinus beats will always intervene between manifest extrasystoles. These extrasystolic discharges are not electrocardiographically manifest, even though the bigeminal rhythm is continuous; the condition was, therefore, termed concealed extrasystoles occurring as a concealed bigeminy.

This postulate of concealed bigeminy was reinforced by the recognition of an analogous phenomenon - that of concealed trigeminy (Schamroth and Marriott, 1963). When manifest ventricular extrasystoles occurred after every two sinus beats (a form of trigeminy) and then apparently lapsed into a haphazard distribution, it was noticed that the intervening sinus beats were always in numbers of $5,8,11$, and 14. This is illustrated in Case 6.

Case 6. Fig. 8 is a section of a continuous recording. The sinus beats between extrasystoles only occur in numbers of $2,5,8,11$, and 14 .

These figures have an analogous significance to the invariable odd numbers seen in cases of concealed bigeminy; namely, that the trigeminal dis- charge was continuous but that one or more consecutive impulses failed to invade the myocardium, i.e. a concealed trigeminy. The situation is illustrated in Fig. 9: beats labelled "a", " $c$ ", and "d" are manifest extrasystoles preventing the descent of sinus beats 3,9 , and 12 ; " $b$ " is a concealed extrasystole, permitting the descent of sinus beat 6 . There are thus 5 sinus beats (labelled 4 to 8) between extrasystoles " $a$ " and " $c$ ". If extrasystole " $c$ " were also concealed, there would then be 8 sinus beats (labelled 4 to 11) between extrasystoles " $a$ " and " $d$ ". With each additional consecutive concealed extrasystole, the number of intervening sinus beats will increase by 3 . Concealed trigeminy is thus recognized when the number of sinus beats occupying interectopic intervals is invariably 2 in excess of a multiple of 3 : expressed mathematically by the formula $3 n+2$ (where $n$ can be any number).

Both concealed bigeminy and trigeminy indicate that the extrasystolic discharge is a rhythmic, continuous, and persistent event; for if there was even an occasional intermission of the rhythmic discharge, 


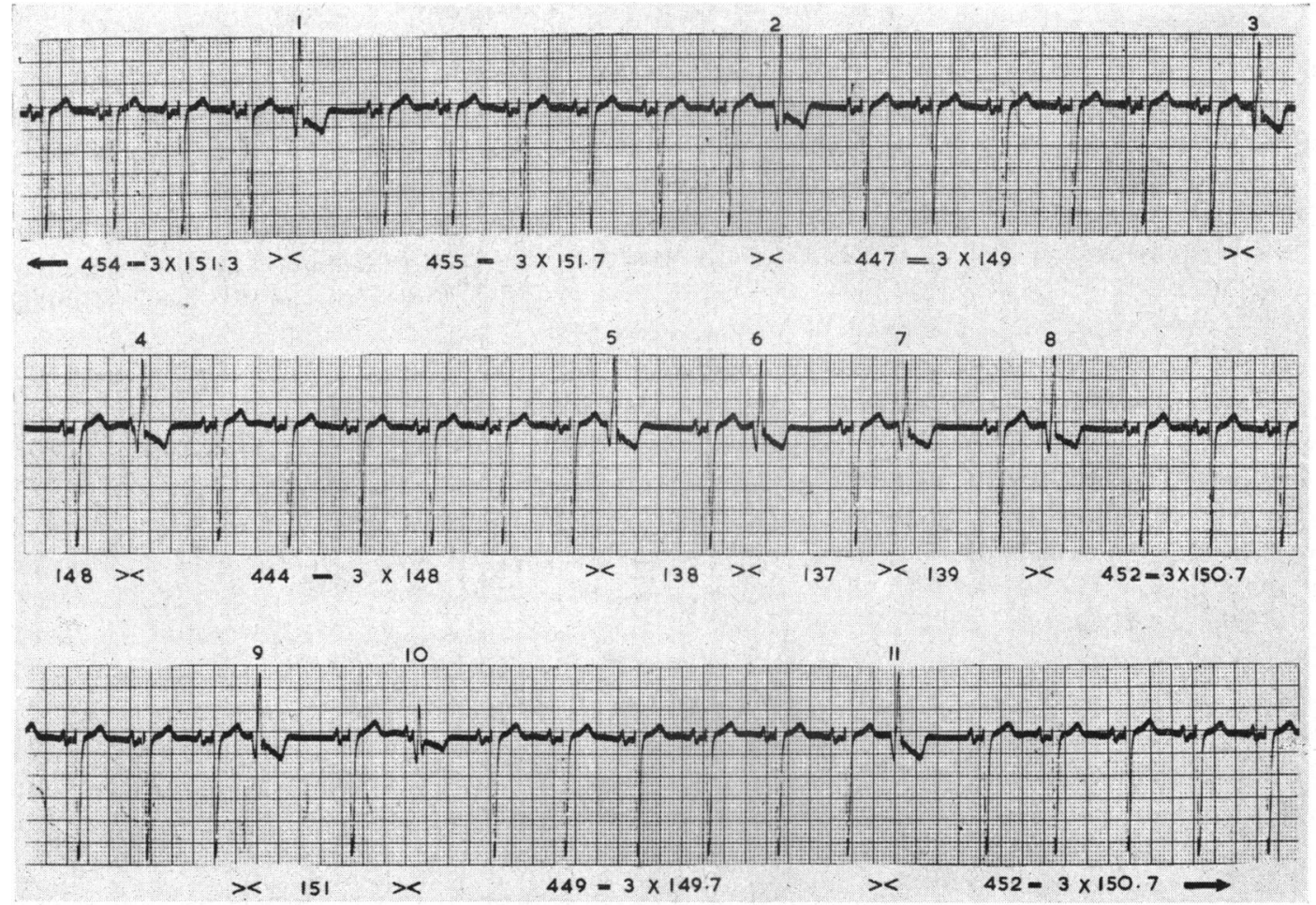

FIG. 10.-Electrocardiogram (continuous strip) showing intermittent parasystole. (See text.)

the pattern would not conform with any degree of consistency to the mathematical sequences outlined above.

(3) Relationship to Parasystole. The relation between parasystolic and extrasystolic impulse formation has, in the past, been uncertain and ill defined. The observed associations have been few and the postulated links often misleading. Thus, Kaufmann and Rothberger (1922) originally postulated that ventricular extrasystolic bigeminy was parasystolic. This tenet, however, was properly dismissed (Mobitz, 1923; Scherf, 1924), because it was based on the presence of constant interectopic intervals which were simply an expression of regular sinus rhythm - the constancy of coupling intervals inevitably leads to constant interectopic intervals when regular sinus rhythm prevails.

There are nevertheless more convincing observations of parasystolic and extrasystolic relationship. Thus, when parasystole is intermittent, in all cases reported to date, each run of parasystolic rhythm has been initiated by an extrasystole (Scherf and Boyd, 1950; Mueller and Baron, 1953; Langendorf and Pick, 1955; Katz and Pick, 1956; Scherf et al., 1957). Conversely, the same ectopic centre that gives rise to a parasystolic rhythm can, at other times, give rise to extrasystolic rhythm (Scherf and Schott, 1930; Vedoya and Rodriguez Battini, 1939; Scherf et al., 1957). Recently, several more cases were reported (Schamroth and Marriott, 1961, 1963), where there was clear evidence of alternate extrasystolic and parasystolic impulse formation from the same ectopic focus. The rhythm was seen to fluctuate repeatedly in the same recording; the same focus that discharged as a parasystolic rhythm could, without interruption, change to extrasystolic bigeminal rhythm that would persist for a few seconds or longer and then revert again to parasystolic rhythm. These features are illustrated in Cases 7 and 4.

Case 7 (Fig. 10). The basic sinus rhythm is interspersed with ectopic beats (numbered 1 to 11 ). These ectopic beats are all of identical contour except complex 10 which is intermediate in configuration between that of the sinus and ectopic beats and is thus a fusion beat. The interectopic intervals between complexes 1 to 5 , and 8 to 11 , are all multiples or near multiples of 150 . These complexes also have variable coupling intervals. The presence of variable coupling intervals, inter- 


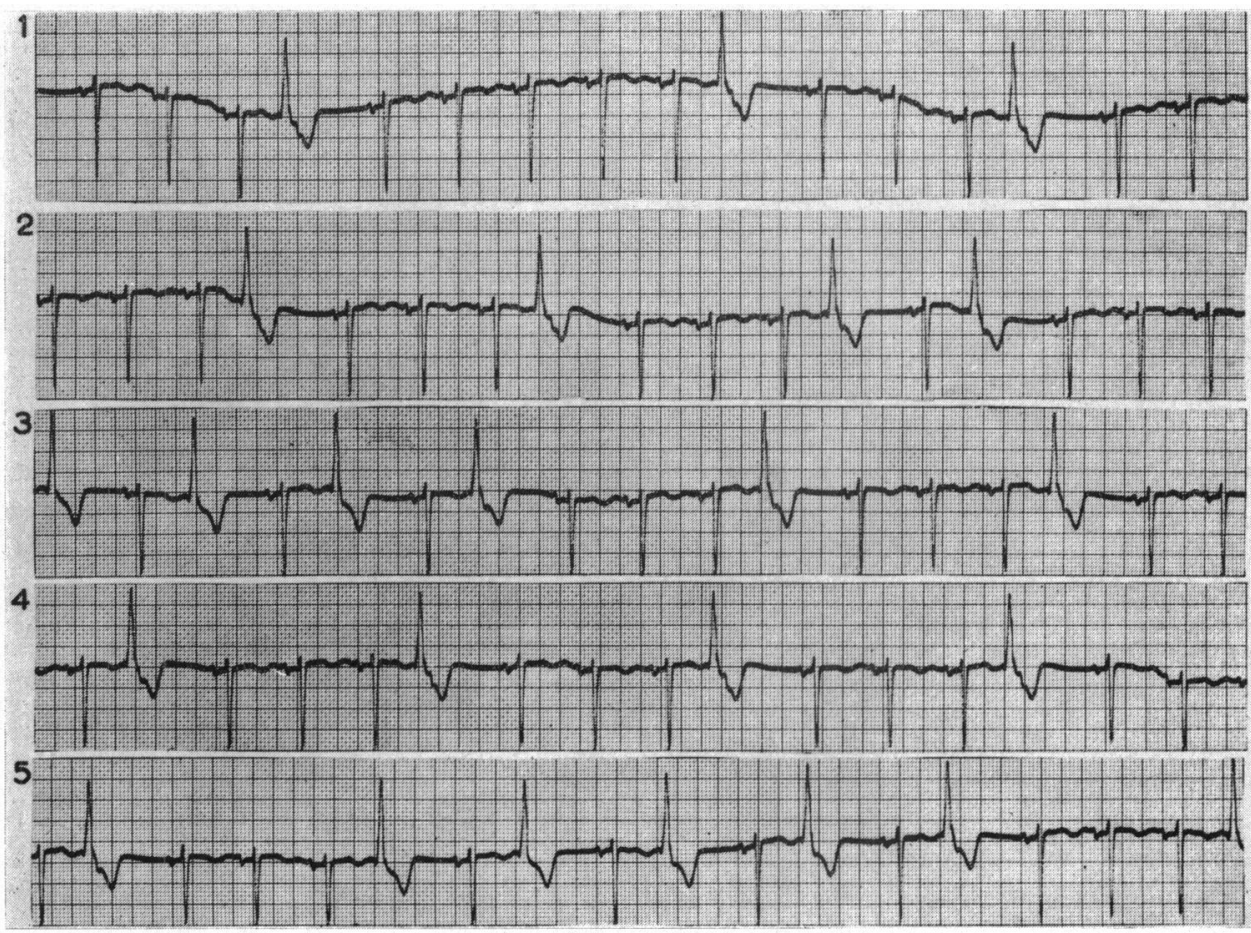

FIG. 11.-Electrocardiogram (continuous strip) of Case 4 showing concealed bigeminy. There are only odd numbers of sinus beats between the manifest extrasystoles.

ectopic intervals with a common denominator, and fusion beats indicates a parasystolic rhythm. The interectopic intervals between complexes 5 to 8 measure about 138 or approximately double the sinus cycle length. The coupling intervals of complexes 6,7 , and 8 are constant. These features indicate that beats 6 to 8 are true extrasystoles. This tracing demonstrates the presence in one recording of parasystole (automatic beats) and extrasystoles (forced beats). The parasystolic rhythm always begins with a forced beat or extrasystole; the ectopic focus then assumes the property of automatic impulse formation and the rhythm changes to parasystole.

This intermittent parasystole could be demonstrated many times during the recording as the rhythm alternated between extrasystolic bigeminy and parasystole. An analysis of all the interectopic intervals during this six-minute recording showed that they could be divided into two main groups as follows.

(A) 73 interectopic intervals that were multiples of 150 (mean 150.35 , range 144.1 to 155.5 but with close grouping around the mean): these represent parasystolic rhythm.

(B) 41 interectopic intervals that were multiples of 136 (mean 133 to 137). These intervals approximate to double the sinus cycle length and represent episodes of extrasystolic rhythm.

Case 4. Fig. 5 (described in the section on parasystole) shows ventricular parasystole. An electrocardiogram recorded the following day (Fig. 11) shows ventricular extrasystoles, in the form of concealed bigeminy, arising from the same ectopic focus. In this patient the extrasystolic and parasystolic rhythms came and went day by day during a 24-day observation period.

Eight further examples have been observed where the rhythm was seen to fluctuate between parasystole and manifest or concealed extrasystolic bigeminy.

If the discharge of a single ectopic ventricular focus can fluctuate repeatedly and without interruption from a parasystolic (automatic) rhythm to an extrasystolic (forced) rhythm-even within the space of a few seconds or minutes-it indicates that the extrasystole represents a forcing or premature precipitation of the parasystolic discharge by the sinus beat, i.e. the pacemaking property that is operative during parasystolic rhythm is also operative during extrasystolic rhythm, but is somehow modified or influenced by the preceding sinus 


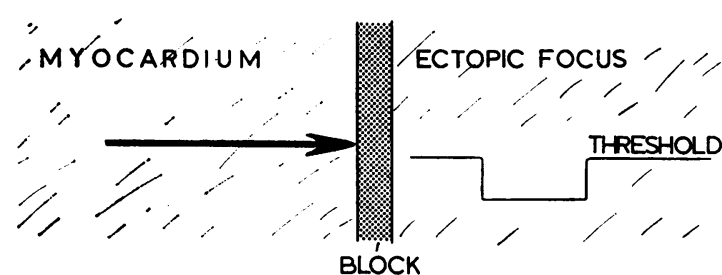

FIG. 12.-Diagrammatic representation of Wedensky facilitation and its application to the precipitation of extrasystoles. (See text.)

beat resulting in its earlier discharge. Furthermore, the same ectopic focus which is protected during parasystolic rhythm must also be protected during extrasystolic rhythm (otherwise the extrasystolic rhythm would obviously be abolished). Thus, the sinus impulse cannot penetrate into or abolish the ectopic discharge but can nevertheless modify the discharge by precipitating it prematurely.

(4) Precipitation or Forcing of Extrasystoles. The development of automatic and forced discharges from the same focus adds to the many cogent arguments already advanced against the re-entry theory (Scherf and Schott, 1953) and makes it quite untenable. However, the theory of ectopic en- hancement-mediated via Wedensky facilitationis easily applicable to an extrasystolic focus having the properties of pacemaking and protection.

It will be recalled that if a sinus impulse is unable to penetrate an ectopic focus the excitability of the focus itself will be enhanced by the mechanism of Wedensky facilitation. The enhancement may be due to either a temporary lowered threshold or a temporary increase in excitability; whatever its mechanism, it may, for convenience, be viewed as a temporary trough or lowered threshold-a temporary enhancement zone. If the ectopic focus is protected, the sinus impulses will be blocked at the periphery of the focus and, on the principles of Wedensky facilitation, this will result in temporary enhancement of the ectopic focus-the development of an enhancement zone or trough of lowered threshold (Fig. 12). And when the unstable slope of slow diastolic depolarization of the ectopic pacemaking cell enters this enhancement zone, its impulse is prematurely precipitated (Fig. 1E-the impulse is precipitated at position 1 instead of 2).

The frequency of extrasystolic precipitation and its rhythmic sequence will depend upon the angle of the slope of diastolic depolarization. If the gradient of the slope is such that it enters the enhancement zone of every alternate sinus beat

A
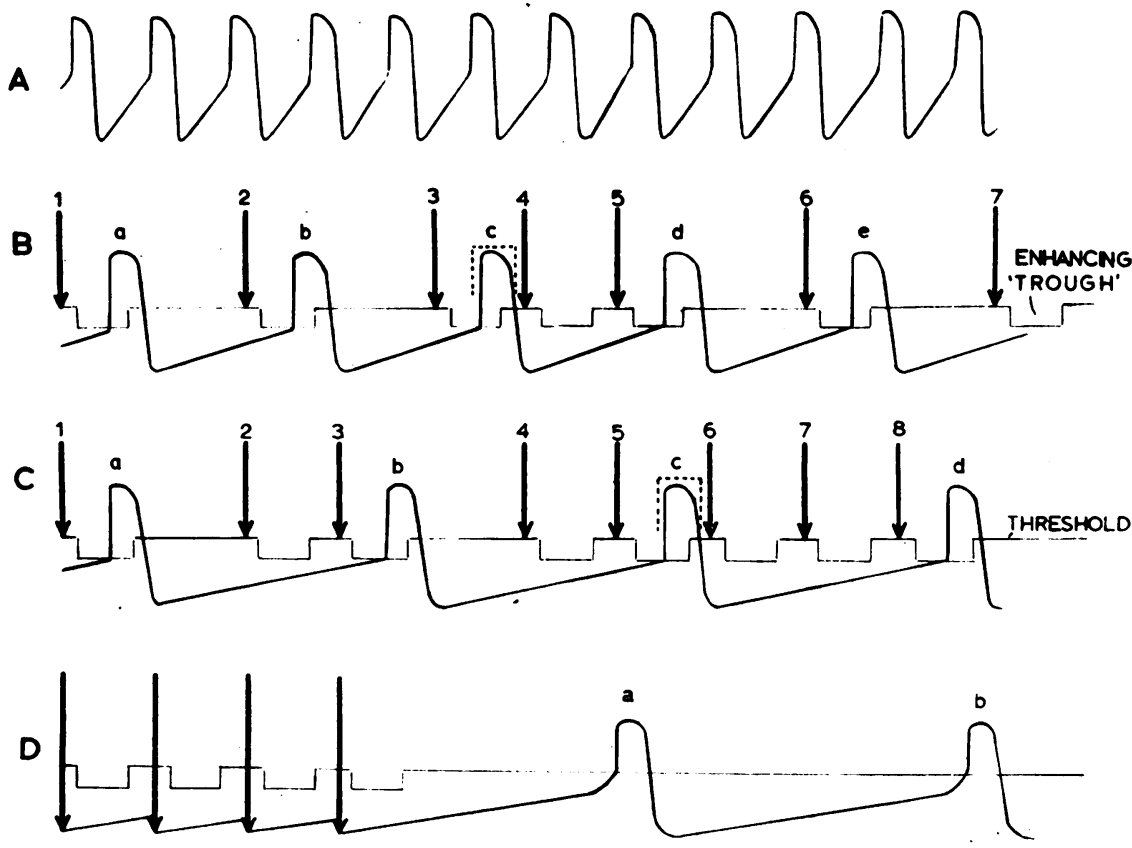

FIG. 13.-Diagrammatic representation of the transmembrane potential in: (A) ventricular tachycardia; (B) bigeminal rhythm; (C) trigeminal rhythm; (D) escape rhythm. (See text.) In escape rhythms the sinus impulses (arrows) penetrate into and abolish the pacemaking discharge prematurely. Impulses labelled "c" are concealed extrasystoles. 

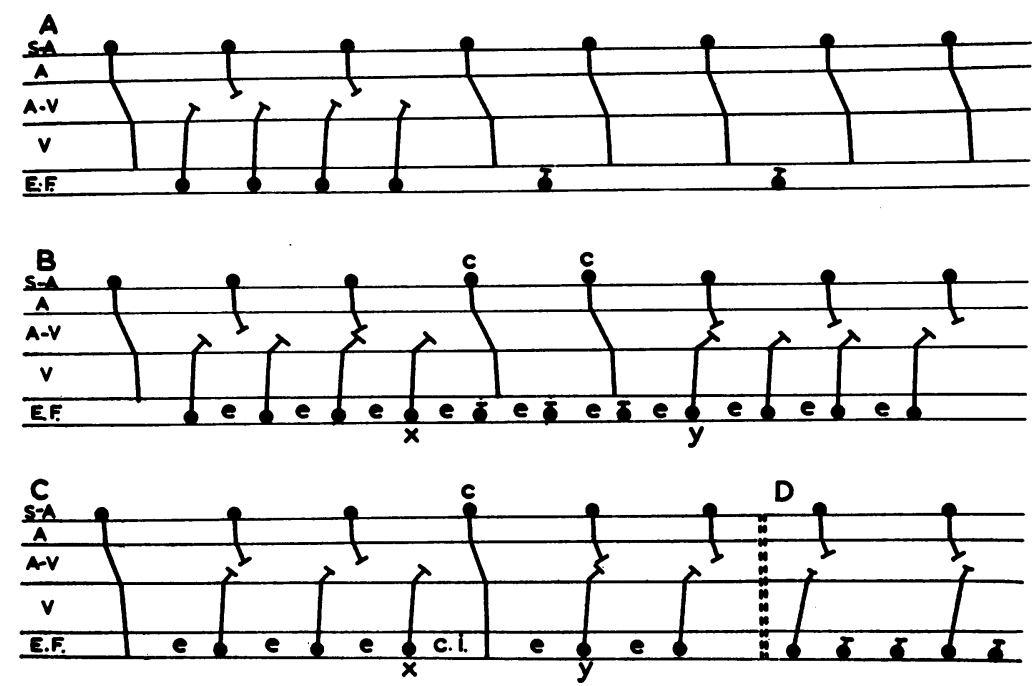

FIG. 14.-Diagrammatic representation of: (A) extrasystolic ventricular tachycardia; (B) parasystolic ventricular tachycardia; (C) idioventricular tachycardia; and (D) ventricular tachycardia with 3:1 exit block. (See text.)

extrasystolic bigeminal rhythm will ensue (Fig. 13B); if the gradient is shallower so that the slope only enters the enhancing zone of every second sinus beat, extrasystolic trigeminal rhythm will result (Fig. 13C). Departures from anticipated sequences, i.e. combinations of bigeminal and trigeminal sequences are explained on the basis of a fluctuating gradient. If the gradient of the slope increases so that the ectopic cycle becomes less than the sinus cycle, ventricular tachycardia ensues (Fig. 13A).

More than one ectopic focus may develop the property of protection. In such circumstances, it is not only the sinus impulse that can enhance and precipitate the ectopic discharge, but the ectopic impulses themselves which, upon arrival at the periphery of the focally blocked areas, will enhance and precipitate each other's activity, thereby resulting in multifocal ventricular extrasystoles.

\section{Ventricular Tachycardia}

Ventricular tachycardia is caused by 3 or more rapid and consecutive discharges from an ectopic ventricular focus. This arrhythmia has been conveniently regarded as a series of 3 or more consecutive extrasystoles. There are, however, indications that not all ventricular tachycardias are extrasystolic in type and that there may, in fact, be more than one mechanism giving rise to this arrhythmia. The possibilities are considered below.

Extrasystolic Ventricular Tachycardia. Extrasystolic ventricular tachycardia always begins with an extrasystole and may be correctly viewed as a series of 3 or more consecutive extrasystoles. Sinus rhythm prevails between paroxysms and subsequent paroxysms again begin with an extrasystole. The interectopic intervals between paroxysms are not in multiples of the ectopic cycle length-indicating the absence of a parasystolic mechanism. Between paroxysms, the sinus rhythm is often interspersed with isolated extrasystoles from the same ectopic focus; and it is noteworthy that there are often only odd numbers of sinus beats between these extrasystoles, thereby indicating the presence of concealed bigeminy. The arrhythmia is schematically represented in A of Fig. 14. The ectopic focus is protected and thus the sinus rhythm does not penetrate into the level of the ectopic focus (E.F.). The first sinus beat is followed by a series of 4 consecutive ectopic beats. This is followed by sinus rhythm during which concealed extrasystoles occur. The first extrasystole and the concealed extrasystoles all have the same coupling interval. These features are illustrated in Case 8.

Case 8. Fig. 15 illustrates a paroxysm of extrasystolic ventricular tachycardia. Isolated extrasystoles from the same ectopic focus are seen interspersed during the sinus rhythm. The paroxysms begin with an extrasystole having the same coupling interval as the isolated extrasystoles. There are only odd numbers of sinus beats in the intervals between extrasystoles.

The extrasystolic ventricular paroxysms thus 


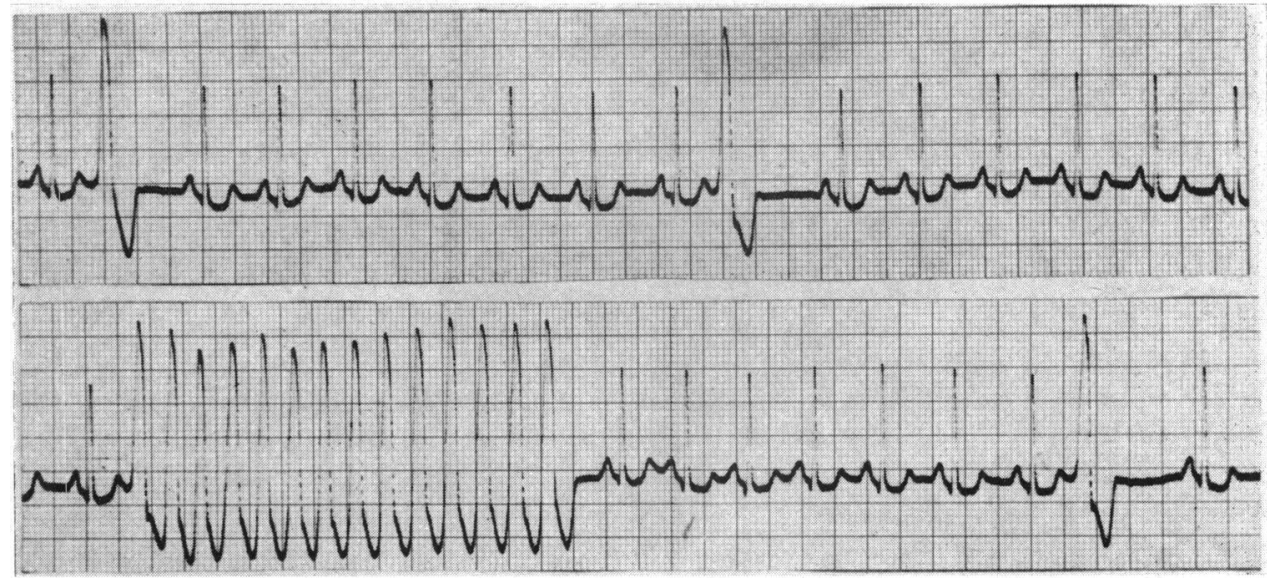

FIG. 15.-Electrocardiogram (continuous strip) showing extrasystolic ventricular tachycardia. (See text.)

arise from a manifest extrasystole occurring during concealed bigeminy. The ectopic focus giving rise to this form of ventricular tachycardia, therefore, enjoys the same properties as the focus giving rise to concealed or manifest extrasystolic bigeminy, i.e. it is a pacemaking centre and is protected from the sinus rhythm.

Parasystolic Ventricular Tachycardia. Parasystolic ventricular tachycardia is a rare arrhythmia. Like extrasystolic ventricular tachycardia it always begins with an extrasystole. However, the interectopic intervals between paroxysms are in simple multiples of the ectopic cycle length. This indicates that the tachycardia continued during the apparent intermission but was not manifest due to the presence of an exit block. The arrhythmia is schematically represented in Fig. 14B. The ectopic focus is protected and thus the sinus beats do not penetrate into the level of the ectopic focus (E.F.). Sinus impulses labelled " $c$ " are consecutive capture beats. The interectopic interval containing these capture beats (interval $X-Y$ ) is a simple multiple of the ectopic cycle length (labelled " $e$ "), i.e. $X-Y=4 \times$ e. Note that the second manifest paroxysm does not commence with an extrasystole but at a moment terminating an interval which is a simple multiple of the ectopic cycle length. Examples of this arrhythmia have been published by Vedoya and Rodriguez Battini (1939); Katz and Pick (1956) (their Figures 194, 196, 214); and Scherf and Bornemann (1961).

Idioventricular Tachycardia. The inherent rate of an idioventricular escape rhythm is slow and, depending upon the site of the pacemaker, usually ranges from 30 to 50 beats per minute (Case 1-
Fig. 2). Nevertheless, it is evident that, under abnormal conditions, this idioventricular rhythm can accelerate. For example, in Case 2 (Fig. 3) the idioventricular escape rhythm occurs at a rate of 82 a minute. Should the ectopic excitability be so enhanced that its rate exceeds the prevailing sinus rate, it becomes dominant, manifesting as an ectopic tachycardia. Idioventricular tachycardia is thus an accelerated idioventricular escape rhythm. Accelerated idioventricular rates are not of necessity very fast, as the ectopic rate need only just exceed the sinus rate to become manifest. Owing to this relatively slow ectopic rate, the opportunities for capture beats are frequent; and it is the effect of the capture beat on the basic ectopic rhythm that distinguishes this form of ventricular tachycardia from the extrasystolic and parasystolic forms. In idioventricular tachycardia, a capture beat will disturb the fundamental ectopic rhythm, i.e. following a capture beat, the ectopic rhythm does not begin again with an extrasystole or at an interval conforming with the parasystolic discharge; it recommences with the inherent escape interval, i.e. the ectopic cycle length. In other words, the ectopic interval including the capture beat is not a simple multiple of the ectopic cycle length. The situation is schematically represented in Diagram C of Fig. 14: ectopic ventricular rhythm occurs at a relatively slow rate (ectopic cycle labelled " $e$ "). The capture beat " $c$ " penetrates the ectopic focus (level E.F.) and discharges its impulse prematurely. The ectopic tachycardia begins again after an interval equal to the ectopic cycle length. The interectopic interval containing the capture beat is composed of the short capture interval (c.i.) and the escape interval (e) and is therefore not a simple 


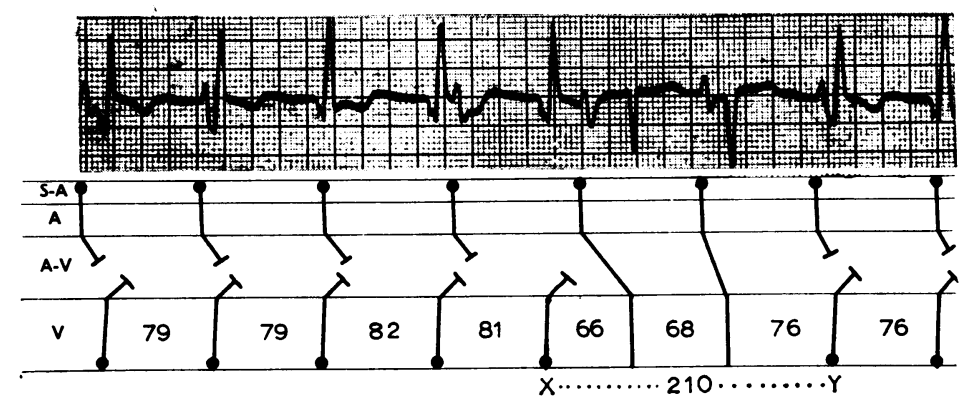

FIG. 16.-Electrocardiogram showing idioventricular tachycardia. (See text.)

multiple of the ectopic cycle length. These features are illustrated in Case 9.

Case 9. Fig. 16 shows a relatively slow ectopic ventricular tachycardia (first 5 bizarre QRS complexes). The ectopic cycle is irregular (the R-R intervals throughout the tracing ranged from 75 to 81). The 6th and 7th QRS complexes are normal and represent consecutive capture beats. The ectopic rhythm begins again after an interval of 75 . The long interectopic interval including the 2 capture beats (labelled $X-Y$ ) measures 210 which is not a simple multiple of 75 to 81 -the ectopic cycle length. Numerous capture beats were seen in this tracing and the ectopic rhythm always began again after an interval ranging from 75 to 81 . There was no instance of a long interectopic interval that included a capture beat being a simple multiple of the ectopic cycle length.

Penetration of the capturing impulse into the ectopic focus (as evidenced by the dislocation of the ectopic rhythm) indicates that the ectopic focus does not have the property of protection. This form of ventricular tachycardia is therefore a simple acceleration of the inherent ventricular escape rhythm-a situation analogous to the simple acceleration of an inherent $A-V$ nodal rhythm found in non-paroxysmal A-V nodal tachycardia (Pick and Dominguez, 1957).

A mechanism contributing to the manifestation of ventricular tachycardia is dissipation of a preexisting exit block. The presence of a regular highgrade exit block will, as in parasystole, mask the fast rate of a ventricular tachycardia. The situation is schematically presented in Fig. 14D. Ventricular tachycardia is present with a 3:1 exit block which masks the rapid ectopic discharge. This principle is illustrated in Case 3 (Fig. 4). A short paroxysm of ventricular tachycardia (third strip) occurs during the slow idioventricular escape rhythm. The paroxysm shows progressive acce- leration within the space of the 4 rapid beats, indicating a Wenckebach conduction sequence at the ectopic ventricular junction. The duration of the paroxysm of 4 beats is exactly that of the idioventricular ectopic cycle length (allowing for the Wenckebach acceleration). This indicates that a 4:1 exit block was present during the slow idioventricular escape rhythm which was momentarily dissipated during the paroxysm. Note that the paroxysm is not extrasystolic or parasystolic, as it does not begin with an extrasystole, and (as indicated in the section on escape rhythm) the capture beat following the paroxysm dislocates the ectopic rhythm, thereby indicating the absence of protection for the ectopic focus.

Dissipation of an exit block may be contributory to the manifestation of any form of ventricular tachycardia.

\section{Ventricular Fibrillation}

Electrophysiologically, the right and left ventricles together act as a single chamber. In ventricular fibrillation the fibres of the ventricular chamber are out of phase with one another and contract independently and asynchronously. The chamber is thus fragmented into numerous irregular areas of excitable, refractory, and partially refractory, tissues; its unitary function is consequently destroyed. The initiation of this abnormal state is favoured by the coincidence of two fundamental events, i.e. (1) uneven recovery of the myocardial chamber; and (2) early impulse formation.

With uneven recovery, the myocardial chamber is divided into irregular zones of refractory, partially refractory, and non-refractory tissue. If, in such circumstances, an early impulse is introduced into, or initiated within, the chamber, it can only activate the non-refractory and partially refractory zones. These activated zones will, in turn, stimulate adjoining tissues as they recover. The fibres of the chamber are thus set further out of phase, the 
fragmentation is accentuated, and the situation rapidly develops into the chaotic state of fibrillation.

Early impulse formation is essentially a succession of rapid beats, as exemplified by ventricular tachycardia and successive paired extrasystoles occurring in rapid succession. The situation is facilitated by rapid impulse formation in several ectopic centres. These conditions favour the development of ventricular fibrillation. Thus, Scherf et al. (1950) observed experimentally that a very rapid ventricular tachycardia ( 300 beats or more a minute) frequently preceded fibrillation. And later, Scherf and Schott (1953) concluded that "the primary disturbance in ventricular fibrillation is the rapid impulse formation in several ectopic centres."

\section{CoRrelation AND SyNTHESIS}

On the basis of these properties, all forms of ectopic ventricular rhythm are closely related and

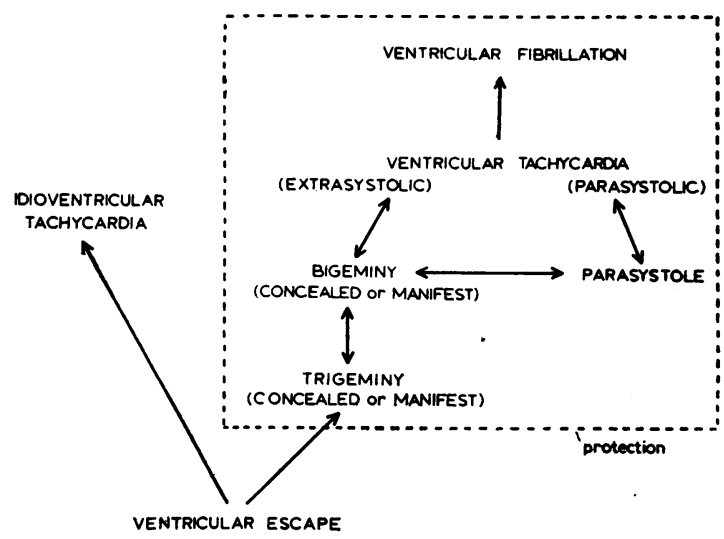

Fig. 17.-Diagram illustrating the genesis and evolution of ectopic ventricular rhythm. Ectopic foci giving rise to the arrhythmias within the dotted square have the property of protection. (See text.)

form part of a common evolutionary process which may be expressed in terms of: (1) the development or acquisition of protection; and (2) an increasing angle to the slope of diastolic depolarization.

Genesis and Evolution of Ectopic Ventricular Rhythm (Fig. 17). Certain ventricular cells-most likely the Purkinje fibres-possess the inherent property of pacemaking function. This pacemaking function is continuously present and is evident as a shallow slope of slow diastolic depolarizing potential. This slow pacemaking centre has no protection from the sinus impulse and its activity is therefore repeatedly abolished by the sinus impulse before it reaches threshold level (Fig. 13D); with suppression of the dominant pacemaker (S-A or
A-V block) the shallow slope is able to reach threshold level and becomes manifest as an escape beat (illustrated as impulse $a$ in Fig. 13D). With prolonged non-arrival of the sinus impulse, the slow ectopic pacemaking activity continues as an idioventricular escape rhythm (illustrated by impulses $a$ and $b$ in Fig. 13D). With an increase in the angle of the slope the rhythm develops into an idioventricular tachycardia.

Progression from escape rhythms is characterized by the acquisition of protection. The ectopic ventricular rhythm cannot now be abolished by the sinus rhythm but is nevertheless precipitated by it, resulting in ventricular extrasystoles. With relatively shallow slopes the rhythm presents as manifest or concealed trigeminy (Fig. 13C). With steeper slopes the ectopic rhythm presents a manifest or concealed trigeminy (Fig. 13B). The rhythm may fluctuate between trigeminy and bigeminy, and complex variations in the angle of the slope may lead to complex extrasystolic distributional patterns.

When the ectopic discharge is completely divorced from sinus influence, it presents as parasystole. The parasystolic focus may periodically come under the influence of the sinus impulse, and the rhythm then fluctuates between parasystole and extrasystolic bigeminy resulting in intermittent parasystole (Fig. 17).

With further progression, the angle of the slope becomes more acute so that eventually the ectopic cycle becomes shorter than the sinus cycle, and ventricular tachycardia ensues. A contributing factor may be the dissipation of a pre-existing exit block.

As the angle of the slope of diastolic depolarization becomes more acute, a very rapid tachycardia results, and this-especially in the presence of multiple ectopic pacemakers-predisposes to ventricular fibrillation.

\section{SUMMARY}

The properties common to all forms of ectopic ventricular rhythm are described and analysed. A unifying concept for the genesis and evolution of ectopic ventricular rhythm is presented. This is based on the principle that the ectopic ventricular focus is a pacemaking centre. Evolution of ectopic ventricular rhythm from its slowest manifestation (escape rhythm) to its fastest manifestations (tachycardia and fibrillation) is dependent upon: (a) the development of protection for the ectopic ventricular focus; and (b) an increasing angle to the slope of diastolic depolarization in the ectopic pacemaking centre. 
This study was assisted by a grant from the Ernest Oppenheimer Memorial Trust, to whose Trustees my sincere thanks are expressed. I am indebted to the Photographic Department, Department of Medicine, University of the Witwatersrand, for the photographic reproductions.

\section{REFERENCES}

Draper, M. H., and Weidmann, S. (1951). Cardiac resting and action potentials recorded with an intracellular electrode. F. Physiol. (Lond.), 115, 74.

Hoffman, B. F., and Cranefield, P. F. (1960). Electrophysiology of the Heart. McGraw-Hill, New York.

Katz, L. N., and Pick, A. (1956). Clinical Electrocardiography. Part 1. The Arrhythmias, p. 147. Lea and Febinger, Philadelphia.

Kaufmann, R., and Rothberger, C. J. (1922). Beiträge zur Entstehungsweise extrasystolischer Allorhythmien. $Z$. ges. $\exp$. Med., 29, 1.

Langendorf, R., and Pick, A. (1955). Mechanisms of intermittent ventricular bigeminy. II. Parasystole, and parasystole or re-entry with conduction disturbance. Circulation, 11, 431.

Mobitz, W. (1923). Über die verschiedene Entstehungsweise extrasystolischer Arhythmien beim Mehschen, ein Beitrag zur Frage der Interferenz mehrerer Rhythmen. Z. ges. exp. Med., 34, 490.

Mueller, P., and Baron, B. (1953). Clinical studies on parasystole. Amer. Heart f., 45, 441.
Pick, A., and Dominguez, P. (1957). Nonparoxysmal A-V nodal tachycardia. Circulation, 16, 1022.

Schamroth, L. (1962). Ventricular parasystole with slow manifest ectopic discharge. Brit. Heart f., 24, 731.

- (1964). The definition of parasystole. Cardiologia (Basel), 44, 37.

-, and Marriott, H. J. L. (1961). Intermittent ventricular parasystole with observations on its relationship to extrasystolic bigeminy. Amer. F. Cardiol., 7, 799.

- and - (1963). Concealed ventricular extrasystoles. Circulation, 27, 1043.

Scherf, D. (1924). Zur Frage der Parasystolic. Wien Arch. inn. Med., 8, 155.

- , and Bornemann, C. (1961). Parasystole with a rapid ventricular center. Amır. Heart f., 62, 320.

- , and Boyd, L. J. (1950). Three unusual cases of parasystole. Amer. Heart f., 39, 650.

—, Morgenbesser, L. J., Nightingale, E. J., and Schaeffeler, K. T. (1950). Mechanism of ventricular fibrillation. Cardiologia (Basel), 16, 232.

- , and Schott, A. (1930). Parasystolie durch einfache Interferenz mit Übergang in Bigeminie. Klin. Wschr., 9, 2191.

mias. William Heinemann, London.

$\longrightarrow,-$, Reid, E. C., and Chamsai, D. G. (1957). Intermittent parasystole. Cardiologia (Basel), 30, 217.

Vedoya, R., and Rodriguez Battini, A. (1939). Un caso de pararritmia mostrando el mecanismo que conduce al bigeminismo extrasistolico. Rev. argent. Cardiol., 6, 313.

Wedensky, N. E. (1903). Die Erregung, Hemmung und Narkose. Pflügers Arch. ges. Physiol., 100, 1. 